\title{
Atmospheric moons Galileo would have loved
}

\author{
Sushil K. Atreya \\ Department of Atmospheric, Oceanic, and Space Sciences, \\ University of Michigan, Ann Arbor, MI 48109-2143, USA \\ email: atreya@umich.edu
}

\begin{abstract}
In the spirit of the symposium and the theme of the session of this presentation, "Our solar system after Galileo, the grand vision," I review briefly a relatively recently discovered phenomenon in the solar system - existence of atmospheres on certain moons, including Io, one of the four moons Galileo discovered four centuries ago. The origin of such atmospheres is discussed, and comparisons are made between various gassy moons.
\end{abstract}

Keywords. Solar system: general, planets and satellites: individual (Enceladus, Ganymede, Io, Jupiter, Moon, Saturn, Neptune, Titan, Triton, Uranus), Sun: abundances

\section{Historical Perspective}

The night of January 8, 1610 was Galileo Galilei's eureka! moment. On this night, Galileo realized that the objects he had observed in close proximity to Jupiter the previous night with his "occhiale" (telescope) were not fixed stars, but were actually the moons of Jupiter, for they had moved and their configuration around Jupiter had changed substantially. With observations during the course of next seven days, Galileo confirmed without any doubt the presence of four moons orbiting Jupiter. Galileo named them the "Medicean Moons," after the Medicis of Florence, and now known as the Galilean Moons. On March 13, 1610, two months after the observations, Galileo published his findings in Sidereus Nuncius that is generally translated as Starry Messenger, or sometimes Starry "Message", apparently referring to Galileo's response to Father Orazio Grassi who had accused Galileo of pretending to be a herald from heaven after seeing the title of the book. With the observations of the moons of Jupiter, Galileo had placed the Copernican hypothesis of heliocentric system on a firm footing. It marked arguably the most monumental turning point for humanity.

With his telescope Galileo went on to reveal a multitude of other mysteries of the heavens, including but not limited to the "seas" and craters on the moon, full planetary phases of Venus and the sunspots not being satellites of the Sun, but no celestial body baffled him so much as Saturn. When Galileo first observed Saturn in 1610, it appeared to him as if the planet was surrounded by two large "moons," giving an appearance of cup handles (Figure 1), unlike anything he had seen at Jupiter. Two years later, he found the moons had disappeared! In 1616, they had taken on the shape of "arms" around Saturn (Figure 1)! With his curiosity, Galileo's frustrations grew, but his twentymagnification telescope lacked the quality for resolving the objects around distant Saturn. Four decades later in 1655, Christiaan Huygens, a Dutch optical physicist and a great admirer of Galileo, finally resolved the mystery using a far better quality telescope what Galileo had observed around Saturn between 1610 and 1616 were disk-shaped rings (Huygens would discover their true shape in 1659). In the same year, 1655, Huygens also discovered a large moon of Saturn, Titan. 


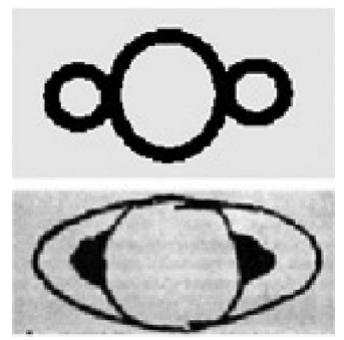

Figure 1. Galileo's observations of Saturn, showing two large "moons" in 1610 (top) and "arms" in 1616 (bottom).

\section{Gases on Io, and lack of them on the other Galilean moons}

Although Galileo's attempts to discover moons around Saturn were unsuccessful and must have been disappointing to him, it is not too outrageous to imagine he must have been convinced of their existence, especially after discovering the moons of Jupiter, and his many attempts at unraveling the mysterious changing nature of the objects around Saturn. He must have wondered of the make-up of the moons of Jupiter and Saturn. Even more important than Galileo's successes and failures at demystifying the solar system is the revolution he had started, by pointing a new way of looking at the solar system, the universe, our place in the cosmos. So, it was only a matter of time before more mysteries of the cosmos would be revealed by more and more innovative means. In this paper, I will attempt to summarize our understanding of one such mystery - the presence of gases around just a handful of the more than one hundred known moons in the solar system. Only Jupiter's moon, Io, Saturn's moon, Titan, Neptune's moon, Triton, have bound atmospheres, whereas Saturn's moon, Enceladus, shows plumes of volatiles (Table 1). In addition, traces of molecules are found near the surfaces of Europa and Ganymede. Since many chapters in this book are devoted to the Galilean moons, here only a brief discussion of their atmospheric aspects is presented.

Unbeknown to him then, Io, one of the moons that Galileo discovered around Jupiter on January 7, 1610, turns out to be the most volcanically active object in the solar system, with over 400 active volcanoes, spewing ashes and gases as high as $500 \mathrm{~km}$ above Io's surface (Figure 2), as revealed first by the Voyager spacecraft nearly four centuries after Galileo's epic discovery of Io. Io's prolific volcanism results from the tidal heat generated within the moon's interior due to its orbital eccentricity. Io's 2:1 mean orbital motion resonance with Europa (Io orbits Jupiter twice for every orbit of Europa) and 4:1 resonance with Ganymede's mean orbital motion maintain a slight eccentricity of 0.0041 in Io's orbit, without which the geologic activity of Io would be minimal. The same orbital resonance causes a slight eccentricity in Europa's orbit also, resulting in tidal heating in the interior of this moon. As a consequence of this heating, models predict possible presence of a salty water ocean beneath Europa's icy crust. Observations by the Galileo spacecraft from 1995 to 2003 revealed an extraordinarily young and smooth surface with few impact craters (Figure 2), which appears to be the result of flows of warm ice from the interior through cracks and fissures in Europa's surface. On the other hand, impact craters are common on Ganymede and Callisto (Figure 2), as these moons experience progressively smaller tidal heating than the two inner Galilean moons Europa and Io. The smaller tidal heating is also one reason why Callisto, Ganymede and Europa have little volatiles above their surfaces. Fueled by incessant volcanism, a tenuous atmosphere of sulfur dioxide with a pressure of approximately a billionth of the Earth's atmosphere (Table 1), or equivalent to that between $110 \mathrm{~km}$ and $160 \mathrm{~km}$ above Earth's surface, is 
formed on Io. However, Ganymede is seemingly a puzzle, especially when compared to Saturn's moon Titan.

The largest of the Galilean moons as well as the largest moon in the solar system, Ganymede, has only traces of hydrogen peroxide (in ice), oxygen and ozone molecules (Table 1), produced by radiolysis of surface ice by high energy charged particles in Ganymede's environment and subsequent chemistry. It is then quite striking that Saturn's moon Titan, which is roughly the same size (Figure 2) and mass as Ganymede (Table 1), is endowed with a massive atmosphere, with even greater pressure than that at the Earth's surface (Table 1). This is despite the fact that Titan was born in a colder part of the solar nebula than Ganymede. In fact, that may have helped Titan. The presence of an atmosphere on the relatively small bodies such as moons depends on a number of interrelated factors, particularly the acquisition, retention, and then the accumulation of the volatiles. The warmer planetesimals that formed Ganymede may not have trapped sufficient quantities of the relevant volatiles to begin with unlike the Titan-forming planetesimals, since the trapping efficiency of certain volatiles (see below) increases at lower

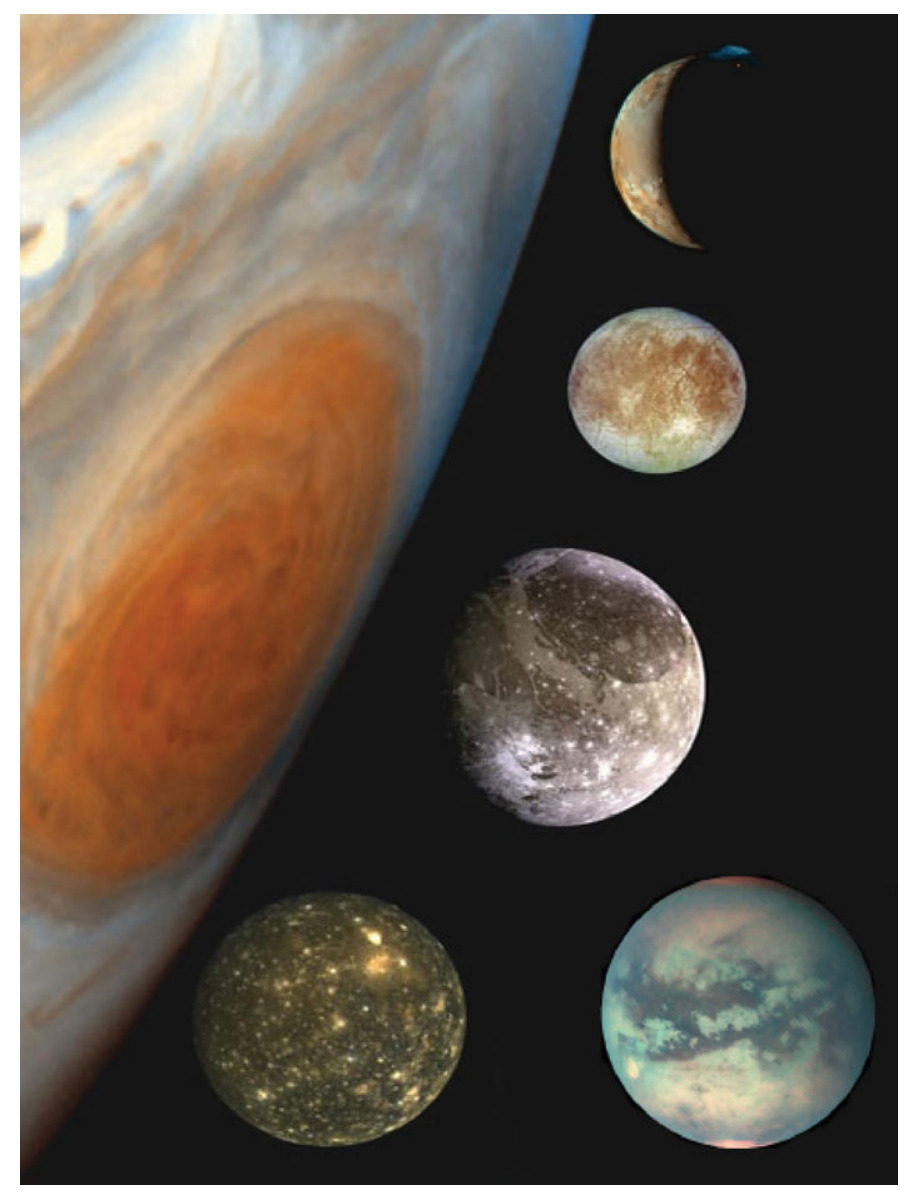

Figure 2. Images of the four Galilean moons alongside Jupiter, together with Saturn's moon, Titan (lower right). Io (top) is the innermost of the four Galilean moons, followed by Europa, Ganymede and Callisto. The image of Io, showing volcanic eruption, was taken by the New Horizons spacecraft during its flyby of Jupiter in 2007, while the images of the other Galilean moons are from the Galileo spacecraft between 1995 and 2003. The image of Titan was taken by the Cassini Visual and Infrared Mapping Spectrometer in 2005. 
Table 1. Key physical, orbital and environmental characteristics of atmospheric moons in the solar system.

\begin{tabular}{|c|c|c|c|c|c|c|}
\hline Characteristic $^{a}$ & Moon & Io & Ganymede & Titan & Enceladus & Triton \\
\hline$R_{\text {planet }}$ & $60.27 R_{E}$ & $5.9 R_{J}$ & $14.99 R_{J}$ & $20.25 R_{S}$ & $3.95 R_{S}$ & $14.33 R_{N}$ \\
\hline$M\left[10^{22} \mathrm{~kg}\right]$ & 7.35 & 8.94 & 14.82 & 13.5 & 0.0108 & 2.14 \\
\hline$R_{e}[\mathrm{~km}]$ & 1738 & 1815 & 2631 & 2575 & 252 & 1352 \\
\hline$\rho\left[\mathrm{kg} / \mathrm{m}^{3}\right]$ & 3340 & 3570 & 1936 & 1880 & 1608 & 2064 \\
\hline$g\left[\mathrm{~m} / \mathrm{s}^{2}\right]$ & 1.622 & 1.796 & 1.43 & 1.35 & 0.11 & 0.78 \\
\hline$T_{s}$ [days] & 27.32 & 1.769 & 7.16 & 15.95 & 1.37 & 5.877 \\
\hline$i$ [degrees] & 5.9 & 0.04 & 0.20 & 0.33 & 0.02 & 157 \\
\hline$e$ & 0.05 & 0.0041 & 0.001 & 0.029 & 0.005 & 0.00 \\
\hline Albedo & 0.12 & 0.63 & 0.4 & 0.2 & $\begin{array}{c}0.99 \text { (bond) } \\
1.37 \text { (geometric) }\end{array}$ & 0.76 \\
\hline$v_{e}[\mathrm{~km} / \mathrm{s}]$ & 2.38 & 2.56 & 2.75 & 2.64 & $\begin{array}{c}0.237 \\
\left(v_{e}<v_{T\left(N_{2}\right)} !\right)^{c}\end{array}$ & 1.50 \\
\hline Surface $T[K]$ & $40-396$ & $130^{b}$ & 110 & 94 & $114-157$ & 38 \\
\hline Surface $P[m b]$ & negligible & $(3-40) \times 10^{-6}$ & negligible & 1500 & $\begin{array}{c}\text { plume } \\
\text { (unbounded) }\end{array}$ & 0.016 \\
\hline Atmosphere & $\begin{array}{c}\text { traces of } \mathrm{H} \\
\mathrm{He}, \mathrm{Na}, \mathrm{K} \\
\mathrm{H}_{2} \mathrm{O}\end{array}$ & $\mathrm{SO}_{2}$ & $\begin{array}{l}\text { traces of } \mathrm{O}_{2}, \\
\mathrm{O}_{3}, \mathrm{H}, \mathrm{H}_{2} \mathrm{O}_{2} \\
\quad \text { in ice }\end{array}$ & $\mathrm{N}_{2}, \mathrm{CH}_{4}$ & $\begin{array}{c}\mathrm{H}_{2} \mathrm{O}, \mathrm{CO}_{2}, \mathrm{CO} \\
\mathrm{CH}_{4}, \mathrm{NH}_{3}, \mathrm{~N}_{2}\end{array}$ & $\mathrm{~N}_{2}, \mathrm{CH}_{4}$ \\
\hline
\end{tabular}

${ }^{a} R_{\text {planet }}$ - mean radial distance between the moon and its parent body ( $E$ : Earth, J: Jupiter, $S:$ Saturn, $N$ : Neptune); $M$ - mass; $R_{e}$ - mean equatorial radius; $\rho$ - mean density; $g$ - equatorial surface gravity; $T_{s}$ - sidereal period; $i$ - orbital inclination; $e$ - orbital eccentricity; $v_{e}$ - equatorial escape velocity.

${ }^{b}$ Io's mean surface temperature. Temperature of some volcanoes, such as the lava eruptions near Pillan Patera can reach as high as $2000 \mathrm{~K}$, much hotter than the hottest basaltic eruptions on Earth, presumably indicating silicate volcanism (McEwen et al. 1998).

${ }^{c} v_{T\left(N_{2}\right)}$ - mean thermal velocity of nitrogen ranges from 0.26 to $0.31 \mathrm{~km} / \mathrm{s}$ for temperatures of $114-157 \mathrm{~K}$ above Enceladus' tiger stripes, indicating that even heavy molecules such as nitrogen can overcome Enceladus' gravity and escape, as $v_{T\left(N_{2}\right)}>v_{e}$, implying that Enceladus cannot maintain a permanent atmosphere in the absence of continuous plumes.

temperatures. Although both Ganymede and Titan had nearly equal probability of retaining the gases, especially the heavier ones such as nitrogen, against escape, whatever little volatiles Ganymede did acquire, were subjected to an irreversible fatal fate, unlike Titan. Unlike Saturn, Jupiter's magnetospheric charged particle environment is intense, severe, and extensive. As a consequence, neither any significant processing to form stable atmospheric gases from the initial inventory of volatiles, nor their accumulation over time could occur at Ganymede, because of charged particle stripping of the atmospheric gases Ganymede might have mustered to form early on. What is then so unique about Ganymede's twin Titan, which succeeded in having a large atmosphere? We will attempt to gain an insight into its possible origin, evolution and fate.

\section{Gases on the Saturnian moon Titan, and plumes on Enceladus}

Molecular nitrogen $\left(\mathrm{N}_{2}\right)$ and methane $\left(\mathrm{CH}_{4}\right)$ comprise the bulk of Titan's atmosphere whose pressure is $1500 \mathrm{mb}$ (Table 1). Nitrogen is approximately $95 \%$ by volume, and methane is roughly $5 \%$. The atmosphere also has trace quantities of heavy hydrocarbons (acetylene, ethane, propane, benzene, etc.), nitriles (such as hydrogen cyanide), and the heavy noble gases - primordial and radiogenic argon $\left({ }^{36} \mathrm{Ar}\right.$ and $\left.{ }^{40} \mathrm{Ar}\right)$ - at parts per million by volume levels or less. In addition, Titan has an extensive haze extending from the lower atmosphere to the ionosphere above $900 \mathrm{~km}$. The heavy hydrocarbon and nitrile molecules are formed by the methane-nitrogen photochemistry in the neutral atmosphere and the ionosphere above it. The same molecules go on to form the haze and soot on Titan (Wilson \& Atreya 2004), similar to the photochemical smog in urban and industrial areas on Earth. However, unlike Earth, haze is key to maintaining the very atmosphere of 
Table 2. Current protosolar elemental abundance ${ }^{a}$, together with elemental enrichment factors at Jupiter and Saturn (updated from Atreya \& Wong 2005).

\begin{tabular}{lccc}
\hline Elements & Sun (protosolar) & Jupiter/Sun & Saturn/Sun \\
\hline $\mathrm{He} / \mathrm{H}$ & 0.09705 & $0.807 \pm 0.02$ & $0.567-0.824^{d}$ \\
$\mathrm{Ne} / \mathrm{H}^{b}$ & $2.10 \times 10^{-4}$ & $0.059 \pm 0.004$ & $?$ \\
$\mathrm{Ar} / \mathrm{H}^{c}$ & $3.62 \times 10^{-6}$ & $2.51 \pm 0.50$ & $?$ \\
$\mathrm{Kr} / \mathrm{H}$ & $2.14 \times 10^{-9}$ & $2.03 \pm 0.38$ & $?$ \\
$\mathrm{Xe} / \mathrm{H}$ & $2.10 \times 10^{-10}$ & $2.11 \pm 0.40$ & $?$ \\
$\mathrm{C} / \mathrm{H}$ & $2.75 \times 10^{-4}$ & $4.30 \pm 1.03$ & $9.3 \pm 1.8^{e}$ \\
$\mathrm{~N} / \mathrm{H}$ & $6.76 \times 10^{-5}$ & $4.90 \pm 1.87$ & $2-4$ \\
$\mathrm{O} / \mathrm{H}$ & $5.13 \times 10^{-4}$ & $0.48 \pm 0.17($ hotspot) & $?$ \\
$\mathrm{~S} / \mathrm{H}$ & $1.55 \times 10^{-5}$ & $2.88 \pm 0.69$ & $?$ \\
$\mathrm{P} / \mathrm{H}$ & $2.57 \times 10^{-7}$ & 1.21 & $5-10$ \\
\hline
\end{tabular}

${ }^{a}$ The protosolar elemental abundances are calculated from the present-day solar photospheric values of Grevesse et al. (2005; Table 1), after accounting for the effects of diffusion at the bottom of the convective zone on the chemical composition of the photosphere, together with the effects of gravitational settling and radiative accelerations, as discussed in Grevesse et al. (2005). The Grevesse et al. (2005) values represent an improvement over the earlier conventional standard compiled by Anders \& Grevesse (1989). The new solar values result from the use of 3D hydrodynamic model of the solar atmosphere, non-LTE effects, and improved atomic and molecular data. The Jupiter results are those measured by the Galileo Probe Mass Spectrometer (GPMS).

${ }^{b}$ Grevesse et al. (2005) derived neon using oxygen as proxy. Direct Ne data from X-ray stars gives 2.7 times greater solar Ne (Drake \& Testa 2005), so that Grevesse et al. (2005) solar Ne/H has been raised by 2.7 , i.e. from $7.76(-5)$ to $2.1(-4)$. Thus, the Jupier/Sun $\mathrm{Ne}=0.059 \pm 0.004$.

${ }^{c}$ Similar to Ne, Grevesse et al. (2005) derived solar argon using oxygen as proxy, which is inappropriate. Therefore, Ar has been changed back to meteoritic (or solar photospheric) value of $3.62 \times 10^{-6}$ (Anders \& Grevesse 1989) in this table.

${ }^{d}$ Based on reanalysis of Voyager data by Conrath \& Gautier (2000). Subject to revision following analysis of Cassini/CIRS (Composite Infrared Spectrometer) data.

${ }^{e}$ Cassini/CIRS measurement (Flasar et al. 2005).

Titan, as discussed later. First, we discuss the origin of the main components of Titan's atmosphere, nitrogen and methane.

Titan's nitrogen: origin, evolution. Nitrogen on a planet or satellite can be primordial or secondary. If primordial, it was delivered as $\mathrm{N}_{2}$ by the planetesimals that formed the object. If it is secondary, it formed from another chemical compound during or subsequent to the accretionary process. Neptune's moon Triton, and Pluto are an example of the former, as both Triton and Pluto formed in the part of the solar nebula where the temperatures were cold enough to trap $\mathrm{N}_{2}$ directly into the icy planetesimals, i.e. below approximately $35 \mathrm{~K}$. That was not the case for Titan, as the temperatures in the subnebula of Saturn where Titan was formed were much too warm to trap $\mathrm{N}_{2}$. This claim was bolstered by the very small amount of primordial argon $\left({ }^{36} \mathrm{Ar}\right)$ detected in Titan's atmosphere by the Gas Chromatograph Mass Spectrometer (GCMS) on the Huygens probe of the Cassini-Huygens Mission (Niemann et al. 2005). Had nitrogen been delivered as $\mathrm{N}_{2}$, Titan should have nearly a million times more ${ }^{36}$ Ar than detected (Niemann et al. 2005; Atreya et al. 2009), as both argon and nitrogen require nearly similar low temperatures for trapping in ice. More specifically, the measured $\mathrm{Ar} / \mathrm{N}_{2}$ was found to be about $2 \times 10^{-7}$ in Titan's present day atmosphere, instead of the solar $\mathrm{Ar} / \mathrm{N}_{2}=0.11$ (Table 2) it would have had if nitrogen was delivered directly as $\mathrm{N}_{2}$ (the $\mathrm{Ar} / \mathrm{N}_{2}$ ratio would have been smaller by a factor of 3-5 in Titan's past compared to that measured today because Titan has lost a large amount of nitrogen via escape during its geologic history). Thus, similar to the nitrogen in the atmosphere of Earth and the other terrestrial planets, Titan's nitrogen atmosphere must be secondary in origin, forming from a compound, most likely ammonia $\left(\mathrm{NH}_{3}\right)$. Ammonia is also the principal reservoir of nitrogen at Saturn and Jupiter where it is found to be greatly enriched relative to the abundance based on the solar nitrogen elemental abundance (Table 2). 


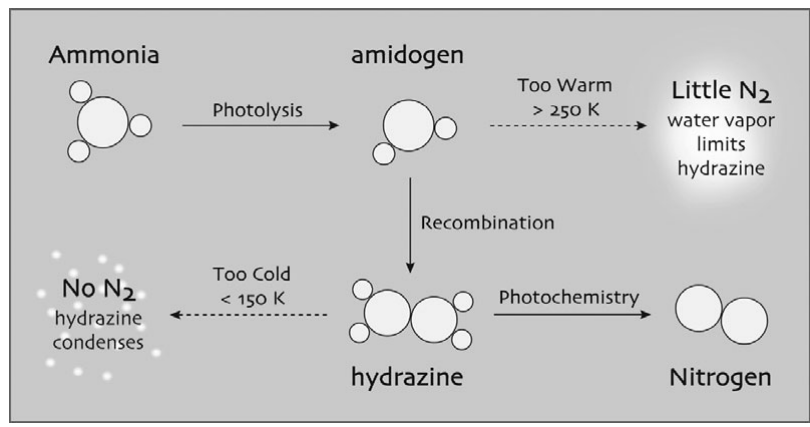

Figure 3. Pathways for production of nitrogen from photochemistry of ammonia.

Nitrogen was detected on Titan first by the Voyager flyby spacecraft in 1979, using the radio occultation technique. Even prior to that various authors had suggested possible presence of nitrogen based on Titan's formation models (review in Atreya et al. 2009). The models suggested ammonia as the source. Atreya et al. (1978) calculated a surface pressure of about 20 bars of $\mathrm{N}_{2}$ on primordial Titan from their pre-Voyager photochemical model of ammonia (Figure 3). Voyager measured a surface pressure of 1.5 bar, which was subsequently confirmed by in situ measurements carried out by the GCMS on the Huygens probe. Titan's present nitrogen is believed to be a relic of a much larger primordial atmosphere in Titan's past as nitrogen has been escaping from the moon over geologic time. It is estimated that Titan started out with 3-5 times more nitrogen, hence a pressure of 5-8 bars of $\mathrm{N}_{2}$ in its primordial past (Niemann et al. 2005), or within a factor of two of Atreya et al.'s predictions. However, photochemical production of nitrogen from ammonia could take place only when Titan was much warmer than today, as the vapor pressure of ammonia is negligible at Titan's current temperature. Models show that Titan was indeed much warmer during its accretionary heating phase. During that time its surface temperature is estimated to have been as high as $400 \mathrm{~K}$, allowing copious ammonia and water vapor to be present in the atmosphere, together with methane. Atreya et al. (1978) and Atreya (1986) show that the most suitable window for photochemical production of nitrogen is between $150 \mathrm{~K}$ and $250 \mathrm{~K}$.

For temperatures greater than $250 \mathrm{~K}$, the saturation vapor pressure of water is appreciable so that reactions between water vapor and intermediate molecules take place that prevent subsequent reactions to continue to completion of nitrogen production (Figure 3). On the other hand, at temperatures below $150 \mathrm{~K}$, although water vapor does not interfere with the ammonia-to-nitrogen chemistry because the $\mathrm{H}_{2} \mathrm{O}$ vapor pressure is much too low, the key intermediate molecule of the ammonia photochemistry, hydrazine $\left(\mathrm{N}_{2} \mathrm{H}_{4}\right)$, condenses, which practically chokes its decomposition by the solar ultraviolet photons, hence the failure to produce nitrogen from it (Figure 3). Using a radiative transfer model of Titan's primordial atmosphere (Adams 2006) and the photochemical chemical scheme of Atreya et al. (1978) and Atreya (1986), it is found that it would take approximately 30 million years to form a 10 bar atmosphere of nitrogen in Titan's past (Adams 2006; Atreya et al. 2009). This is well within the 100 million years or so Titan spent in the above suitable range of temperatures according to evolutionary models of Titan. Another important consideration in the estimate of the time scale for nitrogen production is the solar flux, which was much greater before the Sun reached its main sequence phase at 50 million years, as much as 1000 times greater in the ultraviolet range where the photodissociation of ammonia takes place. This allowed for a more efficient and vigorous production of nitrogen, and was factored into above estimate of time scale. 
Another possible mechanism for the production of nitrogen is an endogenic process. As mentioned previously, Titan's interior must have been warmer early in its geologic history because of accretionary heating. Matson et al. (2007a) found that nitrogen can be formed from thermal decomposition of ammonia in the interior of Enceladus, where high temperatures could result from both tidal heating and radioactive decay (nitrogen is estimated to be less than $1 \%$ in the plumes above Enceladus' tiger stripes, with $\mathrm{H}_{2} \mathrm{O}(90 \%), \mathrm{CO}_{2}(5.3 \%), \mathrm{CO}(4.4 \%), \mathrm{CH}_{4}(0.9 \%), \mathrm{NH}_{3}(0.8 \%)$ and trace molecules comprising the rest, Waite et al. 2009). It should be noted, however, that the gases above Enceladus' surface do not represent a permanent bound atmosphere, i.e. they would be present only as long as the plumes are active (see footnote "c" of Table 1 for details). Matson et al. (2007b) found that endogenic process might be important at Titan also. We estimate as much as 15-20\% of Titan's nitrogen could have been produced by such a process. Since the nitrogen produced in Titan's interior would most likely be stored as clathrate hydrate, it may be released from time to time when the clathrates are destabilized. This would lead to very different nitrogen isotope abundances on Titan than measured by the Huygens GCMS, raising concerns about the validity of the endogenic process (Atreya et al. 2009). Nevertheless, it is potentially an attractive mechanism for producing nitrogen supplementary to that from the inevitable photochemical process, but requires further work. Another idea is that comets supplied ammonia or nitrogen bearing organic molecules from which nitrogen was formed on Titan. However, the nitrogen isotope abundances on Titan do not bear any resemblance to the values in the comets for which the nitrogen isotope abundances are available (Atreya et al. 2009). The hydrogen isotope ratios $(\mathrm{D} / \mathrm{H})$ in Titan's hydrogen and methane molecules are also different, being about one half that in the water vapor of a handful of comets (only four) where it has been measured. However, this isotope ratio may not be a good indicator of the source of Titan's ammonia, since the comparison should really be made with the $\mathrm{D} / \mathrm{H}$ in Titan's surface ice, but such measurements are presently lacking. Moreover, the $\mathrm{D} / \mathrm{H}$ measurement needs to be carried out in many more and diverse comets than the current four from which it is available. Even when the appropriate data for Titan and comets become available, a word of caution about overinterpretation of the data is in order - isotope fractionation occurs on processing of the surface material by cosmic rays, photochemistry or reevaporation-condensation. Hence a true comparison of primordial isotopes may still be lacking. In any event, the cometary contribution to Titan is an idea that also needs to be developed further.

Titan's methane: significance, origin, evolution, cycle. The existence of a nitrogen atmosphere on Titan is critically dependent on the existence of methane gas in the atmosphere. Methane provides the much needed "greenhouse" warming to prevent nitrogen from condensing out of the atmosphere. In the stratosphere, it amounts to as much as $100 \mathrm{~K}$, due to the absorption of the solar infrared flux by haze. Haze results from polymerization and condensation of the photochemical products of methane, particularly, polyynes, polycyclic aromatic hydrocarbons and nitriles in the neutral upper atmosphere (Wilson \& Atreya 2004, 2009) and heavy organics in the ionosphere (Vuitton et al. 2009). In the troposphere, opacity induced by collisions of methane-nitrogen, nitrogen-hydrogen (from methane) and nitrogen-nitrogen molecules results in a net temperature increase of $12 \mathrm{~K}$. Without above heating effects initiated by methane, Titan's atmosphere would shrink substantially due to the condensation of nitrogen.

The origin of methane on Titan is less certain than nitrogen. Methane may have been delivered directly as methane to Titan, or it could have formed on Titan from other carbon bearing entities. The lack of detection of krypton and xenon ( $\mathrm{Kr} \& \mathrm{Xe}$ ) by 


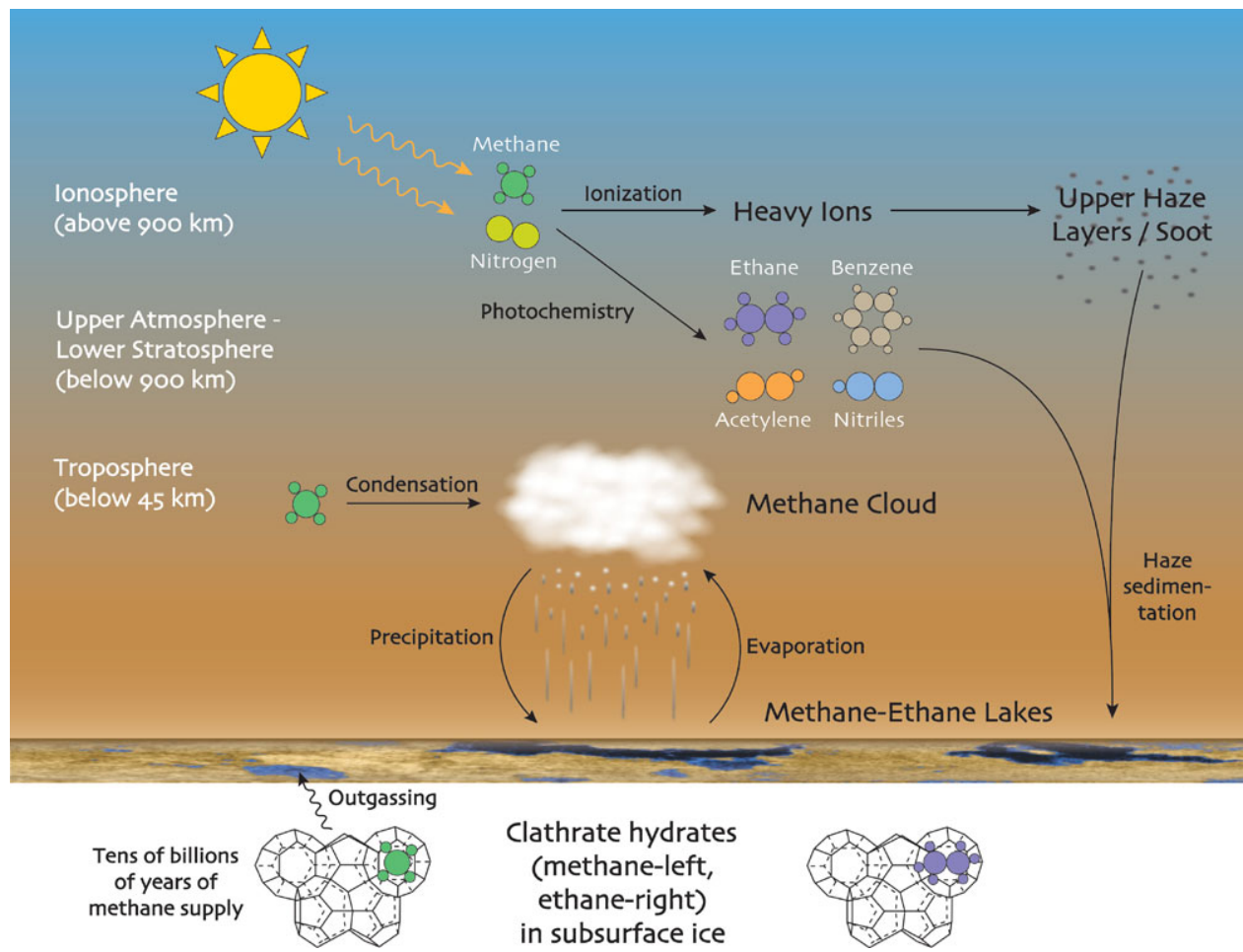

Figure 4. The cycle of methane on Titan. Parts of this figure were inspired by figures in the authors previous publications, including a figure on pp. 48-49 of Atreya (2007a), Figure 1 of Atreya (2007b) and Figure 3 of Lunine \& Atreya (2008).

the Huygens GCMS seems to provide a clue. Had methane arrived at Titan directly as methane, these heavy noble gases would have been found in far greater quantities relative to $\mathrm{N}_{2}$ (see Table 2, column 2) than the upper limit mole fraction of 10 ppbv obtained from the analysis of the Huygens GCMS data since the planetesimals would have trapped these heavy noble gases along with methane. Arguments have been made that the lack of detection of $\mathrm{Kr}$ and Xe may not be indicative of the lack of their existence on Titan. It is suggested that Kr \& Xe may be sequestered as clathrates in the ice below Titan's purported water-ammonia ocean, $50-100 \mathrm{~km}$ beneath the surface. If so, it is somewhat puzzling that they are not released to the atmosphere from time to time, considering that radiogenic argon $\left({ }^{40} \mathrm{Ar}\right)$ is released from the rocks in Titan's core some $2000 \mathrm{~km}$ below the surface and detected in the atmosphere. Suggestions have been made also that $\mathrm{Kr}$ and Xe may be trapped in aerosols. If that were the case, reevaporation of these heavy noble gases would occur as the aerosols descend to the surface, which is $20 \mathrm{~K}$ warmer than the tropopause. Release of Xe and Kr from the surface would also take place upon impacts that occur from time to time. Since the present data pose a dilemma and the models are less than satisfactory, in situ measurements at the surface and below will be required in the future to fully resolve the important question of the origin of methane on Titan.

If methane did form on Titan, it would have done so in a process known as serpentinization (Atreya et al. 2006). Such a process produces methane in terrestrial oceans, at high temperatures $\left(350-500^{\circ} \mathrm{C}\right)$ in Black Smokers, and at relatively mild ones $(30-$ $90^{\circ} \mathrm{C}$ ) in Lost City, some $15 \mathrm{~km}$ from the spreading center on sea floor near the Mid- 
Atlantic Ridge. Serpentinization is a good possibility on primordial Titan also. Today, a 2000-km thick high-pressure ice barrier separates Titan's rocky core from its purported liquid water-ammonia ocean. However, in the past when the temperatures were warmer due to accretionary heat, the ocean is expected to have extended all the way to the core. The water-rock reactions would proceed, hydrogen $\left(\mathrm{H}_{2}\right)$ would be released, and methane would be produced by metal-catalyzed Fischer-Tropsch process involving reactions between $\mathrm{H}_{2}$ and the primordial carbon in the form of $\mathrm{CO}_{2}, \mathrm{CO}$ or carbon grains in the rocks (Atreya et al. 2006). High temperature serpentinization would occur close to the core, and low temperature serpentinization above it with smaller suspended rocky-material. Once produced, methane could be stored as clathrate hydrate $\left(\mathrm{CH}_{4} \cdot 5.75 \mathrm{H}_{2} \mathrm{O}\right)$ in upper ice layers in Titan's interior for subsequent release to the surface. Models indicate that the release of methane from the interior occurs episodically, with the last such episode about half a billion years ago (Tobie et al. 2006).

The surface temperature of Titan is $94 \mathrm{~K}$, which is close to the triple point of methane (and ethane). Once on the surface, methane can remain as liquid. In fact, evidence of numerous wide spread lakes has been found at high northern latitudes $\left(>70^{\circ} \mathrm{N}\right)$ by the Cassini radar (e.g., Stofan et al. 2007) and to a lesser extent in the southern polar region (e.g., Ontario Lacus) where the coverage is limited. Convection allows methane vapor above the surface liquid to rise in the troposphere where it undergoes condensation above approximately $6 \mathrm{~km}$, first as liquid, then changing to ice at colder temperatures at higher elevations (Figure 4). Evidence of both types of clouds is seen in the Cassini orbiter, Huygens probe and ground-based observations. Subsequent precipitation from the clouds would produce methane rain and snow. Thus, the methane meteorological cycle of Titan is similar to the hydrological cycle on Earth (Atreya et al. 2006, 2009; Lunine \& Atreya 2008). For all practical purposes it is a closed cycle, without loss of methane, as any methane removed to the interior from the surface through cracks and fissures is expected to be a temporary local loss as it is likely to be eventually released back out. However, permanent destruction of methane occurs in the stratosphere and the ionosphere.

Irreversible destruction of methane takes place by photochemical conversion of methane to ethane, acetylene, benzene, etc. (Figure 4) in approximately 30 million years (Wilson \& Atreya 2004, 2009), which is a relatively short time in the geologic history of Titan. Thus methane must be released from its storage in Titan's interior from time to time, if Titan were to maintain a relatively stable atmosphere. Despite the photochemical destruction of methane, calculations indicate that Titan's interior may hold tens of billions of years of supply of methane if it was destroyed at today's rate (Atreya et al. 2009). There is no energy crisis on Titan! The principal product of methane photolysis is ethane. As much as $40 \%$ of the methane is expected to be converted to ethane over geologic time (Wilson \& Atreya 2009). Ethane condenses in the upper troposphere of Titan, and ethane clouds have indeed been detected. However, evidence of seas or oceans of ethane predicted in the 1980's is not found. Episodic release of methane could explain why, at least partially. Instead of kilometer deep global ethane oceans, a maximum of only a few meters deep ethane liquid could have formed on Titan's surface since the last episodic release of methane (Wilson \& Atreya 2009). Some of that ethane liquid would become a component of the methane lakes, and is indeed detected in Ontario Lacus (Brown et al. 2008), and some may be sequestered as clathrate in subsurface ice.

Prebiotic molecules. It has been speculated that organics might be present on the Galilean moon Europa, if it accreted from carbonaceous-chondrite type material, or organic molecules formed on Europa if the carbon-containing crust is in contact with 
purported subsurface ocean (Zolotov \& Kargel 2009). Organic molecules have not been detected on Europa, however, either because of their low concentration, oxidation, or destruction by energetic charged particles of Jupiter's magnetosphere. On the other hand, numerous organic molecules have been detected at Titan, and there is even a possibility of the presence of complex prebiotic molecules. The trace hydrocarbons and nitriles produced in gas phase in the upper atmosphere and ionosphere of Titan either go on to form haze or condense in the colder lower stratosphere and at the tropopause $(45 \mathrm{~km}, 74 \mathrm{~K})$. A consequence of this phase and chemical change is that very low abundance of these trace molecules is present in gas phase for detection and quantification in the atmosphere. The remote sensing data from the Cassini orbiter and in situ data from the Huygens probe show that is indeed the case. On the other hand, the same molecules gradually sediment out of Titan's atmosphere, and deposit on to the surface as ices and haze. In time their concentration would build up. The surface thus holds the secrets of the atmosphere and the ionosphere, but the constituents are in far greater concentrations. A slow cooking of the more recent surface deposits by the flux of cosmic rays reaching the ground could result in the formation of complex organic molecules, perhaps even prebiotic molecules. There is even a likelihood that prebiotic molecules were already present in Titan's surface, but without any connection to the atmosphere. In its primordial accretionary period, Titan was most likely above the freezing point of water allowing for the possibility of liquid water. Ammonia and methane must have been abundant in addition to water, and solar ultraviolet flux was substantially greater. In other words, the conditions seem suitable for the formation of prebiotic molecules, as in the Miller-Urey experiment. For these reasons, and the very real likelihood that Titan's interior communicates with the surface, the surface is key to Titan's mysteries (Atreya 2007b).

\section{Conclusion}

Galileo Galilei's paradigm shifting discoveries four centuries ago paved the way for the new discoveries in the solar system, including finding gaseous environments around certain moons. Galileo would be thrilled to learn how far we have come in such a short time, and how rapidly we are moving in finding new solar systems, as he himself did when he discovered a "miniature solar system" on January 7, 1610 from Padova!

\section{Acknowledgement}

Graphics and formatting help provided by Minjin Baek is much appreciated.

\section{References}

Adams, E. Y. 2006, Ph.D. Thesis, University of Michigan, Ann Arbor, USA

Anders, E. \& Grevesse, N. 1989, Geochim. Cosmochim. Acta, 53, 197

Atreya, S. K., Donahue, T. M., \& Kuhn, W. R. 1978, Science, 201, 611

Atreya, S. K. 1986, Atmospheres and ionospheres of the outer planets and their satellites (Berlin, Heidelberg and New York: Springer-Verlag)

Atreya, S. K. 2007a, Scientific American, 296(5), 42

Atreya, S. K. 2007b, Science, 316, 843

Atreya, S. K. \& Wong, A. S. 2005, Space Sci. Revs, 116, 121

Atreya, S. K., Adams, E. Y., Niemann, H. B., Demick-Montelara, J. E., Owen, T. C., Fulchignoni, M., Ferri, F., \& Wilson, E. H. 2006, Planet. Space Sci., 54, 1177

Atreya, S. K., Lorenz, R. D., \& Waite, J. H. 2009, in: R. H. Brown, J.-P. Lebreton \& J. H. Waite (eds.), Titan from Cassini-Huygens (Heidelberg, London and New York: Springer Dordrecht), p. 177 
Brown, R. H., Soderblom, L. A., Soderblom, J. M., Clark, R. N., Jaumann, R., Barnes, J. W., Sotin, C., Buratti, B., Baines, K. H., \& Nicholson, P. D. 2008, Nature, 454, 607

Conrath, B. J. \& Gautier, D. 2000, Icarus, 144, 124

Drake, J. J. \& Testa, P. 2005, Nature, 436, 525

Flasar, F. M. \& the CIRS Team 2005, Science, 308, 975

Grevesse, N., Asplund, M., \& Sauval, J. 2005, in: G. Alecian, O. Richard \& S. Vauclair (eds.), Element stratification in stars, 40 years of atomic diffusion, EAS Publications Series, Vol. 17, p. 21

Lunine, J. I. \& Atreya, S. K. 2008, Nat. Geosci., 1, 159

Matson, D. L., Castillo, J. C., Lunine, J. I., \& Johnson, T. V. 2007a, Icarus, 187, 569

Matson, D. L., Atreya, S. K., Castillo-Rogez, J., Johnson, T. V., Adams, E. Y., \& Lunine, J. I. 2007b, Eos Trans. AGU, 88(52), Fall Meet. Suppl., Abstract P21D-04

McEwen, A. S., Keszthelyi, L., Spencer, J. R., Schubert, G., Matson, D. L., Lopes-Gautier, R., Klaasen, K. P., Johnson, T. V., Head, J. W., Geissler, P., Fagents, S., Davies, A. G., Carr, M. H., Breneman, H. H., Belton, M. J. S., 1998, Science, 281, 87

Niemann, H. B. \& the GCMS Team 2005, Nature, 438, 779

Stofan, E. R., Elachi, C., Lunine, J. I., Lorenz, R. D., Stiles, B., Mitchell, K. L., Ostro, S., Soderblom, L., Wood, C., Zebker, H., Wall, S., Janssen, M., Kirk, R., Lopes, R., Paganelli, F., Radebaugh, J., Wye, L., Anderson, Y., Allison, M., Boehmer, R., Callahan, P., Encrenaz, P., Flamini, E., Franscescetti, G., Gim, Y., Hamilton, G., Hensley, S., Johnson, W. T. K., Kelleher, K., Muhleman, D., Paillou, P., Picardi, G., Posa, F., Roth, L., Seu, R., Shaffer, S., Vetrella, S., West, R. 2007, Nature, 445, 61

Tobie, G., Junine, J. I., \& Sotin, C. 2006, Nature, 440, 61

Vuitton, V., Lavvas, P., Yelle, R. V., Galand, M., Wellbrock, A., Lewis, G. R., Coates, A. J., \& Wahlaund, J.-E. 2009, Planet. Space Sci., 57, 1558

Waite, J. H., Lewis, W. S., Margee, B. A., Lunine, J. I., McKinnon, W. B., Glein, C. R., Mousis, O., Young, D. T., Brockwell, T., Westlake, J., Nguyen, M.-J., Teolis, B. D., Niemann, H. B., McNutt, R. L., Perry, M., \& Ip, W.-H. 2009, Nature, 460, 487

Wilson, E. H. \& Atreya, S. K. 2004, J. Geophys. Res., 109, E06002

Wilson, E. H. \& Atreya, S. K. 2009, J. Phys. Chem., 113, 11221

Zolotov, M. Y. \& Kargel, J. S. 2009, in: R. T. Pappalardo, W. B. McKinnon \& K. Khurana (eds.), Europa (University of Arizona Press), p. 449 